\title{
Preface to the special issue: novel applications of main refractory metals and their compounds
}

\author{
Jin-Shu Wang ${ }^{1}$. Jun Wang ${ }^{2}$ \\ Published online: 16 September 2020 \\ (c) The Nonferrous Metals Society of China 2020
}

Tungsten is an important material widely used not only in traditional refractory applications, but also in new functional areas. Launched in early 2019, Tungsten journal is dedicated to the science and technology of tungsten and related metals, including molybdenum, cobalt, vanadium, tantalum, niobium, rhenium, as well as their alloys and compounds. The present issue consists of eight contributions with five review papers and three original research articles, covering a set of topics such as electron emission of $\mathrm{W}$ materials, energy conversion and storage of $\mathrm{W}$ compounds, microstructure evolutions in rolled Mo alloys, and catalysis performance of $\mathrm{Pd}, \mathrm{Co}$ and $\mathrm{Re}$ nanopartilces.

The special topic focuses on the novel applications of main refractory metals and their compounds. First, Wang et al. reviews the recent advances in thermionic cathode materials, discussing the improvement of electron emissions in tungsten by rare earth elements doping. Zhang et al. then reports the progress on the photocatalytic performance of $\mathrm{WO}_{3}$-based photocatalysts for degradation of volatile organic compounds (VOCs). Lei et al. also summarizes the development of $\mathrm{WS}_{2}$ and its composites for electrochemical energy storage and conversion applications. Besides, one research paper by McEvoy et al. reports a new synthesis method for production of large-area polycrystalline $\mathrm{WTe}_{2}$ films. Moreover, $\mathrm{WTe}_{2}$ has attracted great interests in recent years due to its large non-saturating magnetoresistance. In addition to tungsten-based materials, some articles

Jin-Shu Wang

wangjsh@bjut.edu.cn

1 Key Laboratory of Advanced Functional Materials, Education Ministry of China, Faculty of Materials and Manufacturing, Beijing University of Technology, Beijing 100124, China

2 Laboratory of Micro-Nano Optoelectronic Materials and Devices, Key Laboratory of Materials for High-Power Laser, State Key Laboratory of High Field Laser Physics, Shanghai Institute of Optics and Fine Mechanics, Chinese Academy of Sciences, Shanghai 201800, China consisted of tantalum, molybdenum and rhenium elements are also included in current issue. Wang et al. studies the texture, microstructure, mechanical properties, anisotropy and the correlation of Mo-La plates for the rolled and the annealed states at different temperatures. Guo et al. reviews the crystal growth, magneto-transport measurements and theoretical studies of tantalum dipnictides $\mathrm{TaAs}_{2}$ and $\mathrm{TaP}_{2}$, emphasizing on the discussions of the nontrivial topological states. Hao et al. presents the recent advances on the fabrication techniques and applications of $\mathrm{Pd}$, Co and Re nanopartilces. Finally, a modeling research work on Al-Hf-Nb-Mo-Ta-Ti-V-Zr refractory high-entropy alloys is presented by Shafiei et al.

I hope the contents in this special issue can inspire readers to explore new visions into the applications of main refractory metals and their compounds. More contributions in related areas are highly encouraged to be submitted to the Tungsten journal. Also, I would like to appreciate all the authors, reviewers, editors, and publishing staff for their great efforts and contributions for the publication of this special issue.
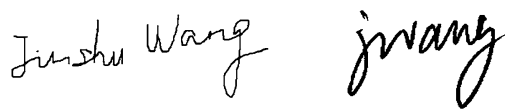

Jin-Shu Wang, Jun Wang

Sep 4, 2020

Publisher's Note Springer Nature remains neutral with regard to jurisdictional claims in published maps and institutional affiliations 


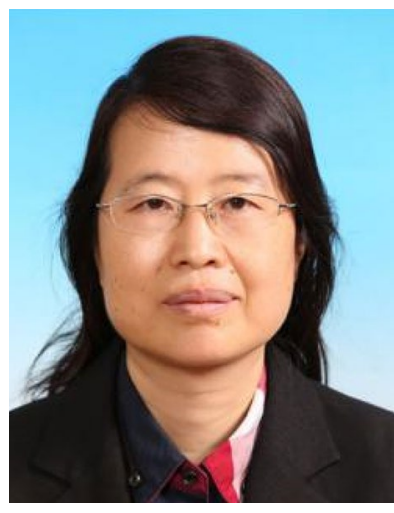

Dr. Jin-Shu Wang is a professor of Materials Science and Engineering in Beijing University of Technology (BJUT). She received her $\mathrm{Ph} . \mathrm{D}$. degree from the College of Materials Science and Engineering, BJUT, in 1999. From 2002 to 2004, she had worked as a postdoctoral researcher in Tohoku University, Japan. Her research interests include refractory metal-based electronic emission materials, renewable energy and environmental materials. She has published over 200 peer-reviewed journal (Adv. Funct. Mater., Appl. Catal. B: Environ., J. Mater. Chem. $A$, etc.) papers and 2 books. She has authorized over 40 invention patents of China and 2 American invention patents. She obtained the National Nature Science Fund for Distinguished Young Scholars in 2012. She was awarded the Distinguished Professor of Chang Jiang Scholars Program by the Ministry of Education, China, in 2015. She is a senior committee member of Vacuum Electron Society, Chinese Electronics Society, a committee member of Chinese Corrosion \& Protection Society, a member of Society of Metallurgical Physicochemistry, and a member of Photocatalysis Industry Association of China. She is also an editor of journals of Powder Metallurgy Technology, Tungsten, Vacuum electronics Technology, etc.

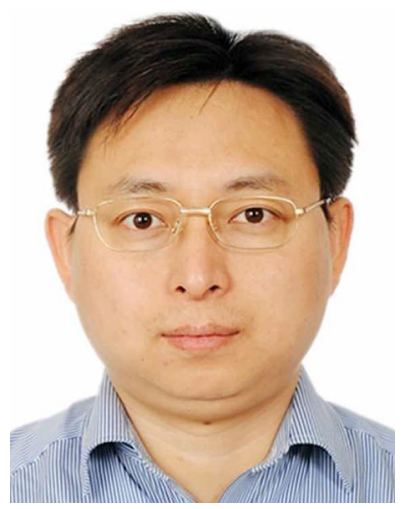

Dr. Jun Wang is a professor with Shanghai Institute of Optics and Fine Mechanics (SIOM), Chinese Academy of Sciences (CAS). He obtained his Ph.D. degree from the Chinese University of Hong Kong in 2006. Then he was granted an IRCSET postdoctoral fellowship and worked at Trinity College Dublin, Ireland. In 2011, he relocated his research activities to SIOM under the financial support from the 100-Talent Program of CAS. In 2018 and 2015, he was awarded by the National

10,000-Talent Program and National Natural Science FoundationOutstanding Youth Foundation, respectively. His research interests are focused on nonlinear optics in low-dimensional materials. So far, he has published more than 160 peer-reviewed SCI journal papers in Nature Nanotech., Prog. Mater. Sci., Adv. Mater., Nature Commun., ACS Nano, Laser Photon. Rev., etc., including 10 ESI papers and has over 6000 citations. 